Behavior

Modification

\title{
Virtual Reality Versus Computer-Aided Exposure Treatments for Fear of Flying
}

\begin{tabular}{|r|l|}
\hline Journal: & Behavior Modification \\
\hline Manuscript ID: & BMOD-10-0045.R2 \\
\hline Manuscript Type: & Original Manuscripts \\
\hline Author: & n/a \\
\hline Komplete List of Authors: & $\begin{array}{l}\text { Tortella-Feliu, Miquel; University of the Balearic Islands, University } \\
\text { Research Institute on Health Sciences } \\
\text { Botella, Cristina; Jaume I University } \\
\text { Llabrés, Jordi; University of the Balearic Islands } \\
\text { Bretón, Juana; Jaume I University } \\
\text { Riera del Amo, Antonio; Jaume I University } \\
\text { Baños, Rosa; University of Valencia } \\
\text { Gelabert, Joan; University of the Balearic Islands }\end{array}$ \\
\hline Keywords: & $\begin{array}{l}\text { fear of flying, virtual reality, computer-aided, treatment, } \\
\text { randomized controlled trial, exposure }\end{array}$ \\
\hline
\end{tabular}

\section{SCHOLARONE ${ }^{\text {tw }}$ Manuscripts}


Running Head: VR AND COMPUTER-AIDED EXPOSURE TREATMENTS

Virtual Reality Versus Computer-Aided Exposure Treatments for Fear of Flying Miquel Tortella-Feliu ${ }^{1 *}$, Cristina Botella ${ }^{2,4}$, Jordi Llabrés ${ }^{1}$, Juana Bretón ${ }^{2}$, Antonio Riera del Amo ${ }^{1}$, Rosa M. Baños ${ }^{3,4}$, and Gelabert, J.M. ${ }^{1}$

${ }^{1}$ University of the Balearic Islands, University Research Institute on Health Sciences (IUNICS)

2 Jaume I University

${ }^{3}$ University of Valencia

${ }^{4}$ CIBER Fisiopatologia Obesidad y Nutricion (CIBEROBN).

\footnotetext{
* Corresponding author: Miquel Tortella-Feliu. Universitat de les Illes Balears. IUNICS. Carretera de Valldemossa km.7,5. 07122 Palma (Mallorca, Spain) E-mail: miquel@ ndpc.cat, Phone (+34)971172579, Fax (+34)971173190.
} 
VR and CAE treatments 2

\begin{abstract}
Evidence is growing that two modalities of computer-based exposure therapies, virtual reality and computer-aided psychotherapy, are effective in treating anxiety disorders including fear of flying; however, they have not yet been directly compared. The aim of this study was to analyze the efficacy of three computer-based exposure treatments for fear of flying: virtual reality exposure therapy (VRET), computer-aided exposure with a therapist's assistance throughout exposure sessions (CAE-T), and selfadministered computer-aided exposure (CAE-SA). Sixty participants with flying phobia were randomly assigned to VRET, CAE-T, or CAE-SA. Results indicate that the three interventions were effective in reducing fear of flying at post-treatment and at one-year follow-up; furthermore, there were no significant differences between them in any of the outcome measure. Large within-group effect sizes were found for all three treatment conditions at both post-treatment and at follow-up. Our results suggest that therapist involvement might be minimized during computer-based treatments, and that CAE can be as effective as VRET in reducing fear of flying.
\end{abstract}

Keywords: fear of flying, virtual reality, computer-aided, treatment, randomized controlled trial, exposure. 
Virtual Reality Versus Computer-Aided Exposure Treatments for Fear of Flying

\section{Introduction}

Many computer-assisted treatment procedures have been developed for use in clinical psychology. In parallel, a surge of research in this new field has provided increasing evidence that several kinds of computer-assisted treatment procedures are highly effective in treating anxiety disorders, especially phobic disorders (Cuijpers, Marks, van Straten, Cavanagh, \& Andersson, 2009; Krijn, Emmelkamp, Olafsson, \& Biemond, 2004; Marks, Cavanagh, \& Gega, 2007a; Parsons \& Rizzo, 2008; Powers \& Emmelkamp, 2008). Depending upon the type of technology used and the main goals for using computers and other electronic devices in psychological treatment, at least two broad categories of computer-assisted treatments have emerged: virtual reality (VR) and computer-aided psychotherapy. We fully recognize, as do other authors (e.g., Marks \& Cavanagh, 2009; Marks et al., 2007a) that the distinction between these categories is fuzzy; however, until recently, research on VR and other computer-based psychological treatments has evolved independently as two separate research paths.

Computer-aided psychotherapy, as defined by Marks et al. (2007a) and Marks, Cavanagh, and Gega (2007b) refers to any computing system that aids face-to-face treatment by using patient input to process data and guide treatment decisions, saving therapist time.

VR uses computer-generated environments to simulate stimuli through multiple sensorial channels (mainly visual and auditory), thereby allowing the user to interact with the virtual world while also achieving a sense of being physically there. 
The main differences between VR and other computer-based treatments are that (a) most of the VR treatments delegate few treatment tasks to the system [with some few exceptions like Botella et al.'s (2008) use of VR in a completely self-administered interned-based treatment for animal phobia)], as do computer aided-psychotherapy, and (b) that VR has been mostly employed as a tool for exposure therapy, called VR exposure therapy (VRET), while computer-aided psychotherapy treatments have been used to deliver several therapeutic strategies.

Computer-aided psychotherapy is usually designed to facilitate several routine interactive therapy strategies, including education and assessment of current problems with feedback and change monitoring, as well as action plans and goals, behavioral experiments or systematic exposure, among others. Methods for accessing content include standalone or networked personal computers, interactive telephone voice response systems, handheld device systems and non-immersive display systems for images and sounds (Marks \& Cavanagh, 2009; Marks et al., 2007a, 2007b). More than one hundred computer-aided psychotherapy systems exist for the treatment of all anxiety disorders, and other psychological disorders and health problems (for a comprehensive review see Marks et al. 2007a). Computer-aided psychotherapy is as effective as face-to-face treatments, at least for anxiety disorders (Cuijpers et al., 2009). Increasing development and use of computer-aided psychotherapy has relied not only in the fact that it saves therapist time by facilitating routine interactive strategies in therapy without compromising efficacy (e.g. Marks, Kenwright, McDonough, Wittaker, \& Mataix-Cols, 2004; McCrone et al., 2007), but also in other multiple benefits. Computer-aided treatments increase patients' access to therapy - in face of help demands exceeding resources to be supplied by qualified therapists - allow home access, ensure confidentiality, reduce patient inhibition and increase his/her motivation 
for treatment, and can quickly report patients' progress. It is also notable that computeraided psychotherapy seems to be helpful in clinician training, as well as in refining process and outcome research in psychotherapy (see Marks \& Cavanagh, 2009; Marks et al., 2007). As Andersson (2009) stated about internet-based therapy, computer-aided psychotherapy is part of the long tradition in cognitive-behavioral therapy of shortening treatment without compromising efficacy, mirroring face-to-face treatments, and facilitating its dissemination and patient access.

Some computer-aided psychotherapy treatments generally resemble VRET. This is the case for direct non-immersive display systems for treating specific phobias and other anxiety disorders, which provide a screen display of anxiety provoking cues (images and sometimes sounds) in order to conduct systematic exposure. However, these kinds of systems do not afford interaction with computer-generated virtual environments as VRET does. Focusing on fear of flying, Bornas and colleagues developed the Computer-Assisted Fear of Flying Treatment (Bornas, Fullana, TortellaFeliu, Llabrés, \& García de la Banda, 2001; Bornas et al., 2001; Bornas et al., 2006), a system that provides exposure to photos and accompanying sounds of flying situations on a personal computer with a standard screen and headphones. In two controlled trials (Bornas et al., 2001, 2006), this computer-assisted exposure (CAE) system was shown to be effective in reducing fear of flying, and CAE alone appeared to have some advantages in terms of efficiency over other multicomponent programs, suggesting that CAE could save time and resources compared to regular face-to-face treatments for fear of flying, but it has not yet been tested as a true self-administered treatment.

As a psychological treatment, VR has been primarily used to provide systematic exposure to anxiety provoking cues by means of immersive computer-generated environments, thereby becoming a computer-based alternative to standard in vivo 
exposure (Powers \& Emmelkamp, 2008). Evidence supports that VRET, which generally includes cognitive restructuring strategies, is effective at treating anxiety disorders, as recently summarized in the meta-analytic studies by Parsons and Rizzo (2008), Powers and Emmelkamp (2008) and Wolitzky-Taylor, Horowitz, Powers, and Telch (2008) as well as in reviews by Anderson, Jacobs, and Rothbaum (2004), Botella et al. (2006) and Krijn et al., (2004) exhibiting effect sizes generally comparable to those achieved through in vivo exposure. VRET has been used especially for reducing fear of flying, and several controlled trials have tested its efficacy for this phobia (Krijn et al., 2007; Maltby, Kirsch, Mayers, \& Allen, 2002; Mühlberger, Herrmann, Wiedemann, Ellgring, \& Pauli, 2001; Mühlberger, Weik, Pauli, \& Wiedemann, 2006; Mühlberger, Wiedemann, \& Pauli, 2003; Rothbaum et al., 2006; Rothbaum, Hodges, Smith, Lee, \& Price, 2000; Wiederhold \& Wiederhold, 2003). In general, these trials indicate that VRET provides clear advantages over no treatment conditions in reducing fear of flying, and it is at least as effective as in vivo exposure. Additionally, VRET has proven to be effective in open trials including those conducted with the "Virtual Flight" environment used in the present study (Baños et al., 2002; Botella, Osma, GarcíaPalacios, Quero, \& Baños, 2004). Beyond efficacy criteria, VRET has a number of advantages over other kinds of exposure treatments, some of which are shared with computer-aided psychotherapy, including confidentiality, patient's perceived security during sessions, higher levels of control of the exposure conditions, and the ability to be fully conducted in a therapist's office.

Computer-based treatment systems have a variety of potential problems to be adressed. These include three closely inter-related features: patient attitudes and expectations towards treatment, attrition rates, and the guidance and support therapists must provide to patients (e.g., Andersson, 2009). Some of these problems are more 
related to clinicians' concerns than with facts. For instance, professional expectations are that patients will find face-to-face therapies more acceptable than computer-aided ones (Whitfield \& Williams, 2004). However, evidence from multiple studies suggests that patients' satisfaction, preferences, and acceptability of computer-aided psychotherapy treatments are high and quite similar to their evaluations of face-to-face interventions (e.g., Cavanagh et al., 2009; Graham, Franses, Kenwright, \& Marks, 2000; Kaltenthaler, Parry, \& Beverley, 2004; Marks, Kenwright, McDonough, Whittaker, \& Mataix-Cols, 2004). The same is true for VRET (e.g., Mühlberger et al., 2006;

Rothbaum et al., 2006). However, other studies do report greater patient satisfaction for face-to-face interventions (e.g., Bowers et al., 1993). This deserves attention because a higher credibility of computer-based treatment appears to be related to better treatment outcomes than other factors like severity of fear of flying or the severity of overall psychopathology (Mühlberger et al., 2006). Furthermore, in computer-aided psychotherapy research, data clearly indicate that, as in any kind of treatment, less satisfaction is associated with greater attrition (Marks et al., 2007a). In general, it can be stated that drop-out rates do not differ across computer-aided psychotherapy and faceto-face care (Cuijpers et al., 2009) and, once again, the same occurs in VRET (Krijn et al., 2004).

Regarding therapist support, research indicates that although computer-based treatments cannot totally replace human interaction, they can reduce face-to-face time. A number of meta-analytic studies have found that computer-aided treatment effects are smaller when patients had less therapist time (Cuijpers et al., 2009; Palmqvist, Carlbring, \& Andersson, 2008). This is an important challenge for computer-based treatments and establishing the best methods for their self-administration. Currently, it is widely recognized that some personal support increases adherence to computer-aided 
psychotherapy, although it can increase costs; furthermore, the best types, frequency, duration and sites of clinician support have not yet well established (Andersson, 2009; Marks et al., 2007a). As described below, in the present study different kinds of computer-based treatments with differing degrees of therapist involvement were delivered; they were then compared in terms of patients' treatment expectations, satisfaction, and adherence.

The aim of this study was to compare efficacy of three computer-assisted exposure-based treatments for fear of flying: (a) virtual reality exposure assisted by a therapist supporting the patients throughout the therapy process and using cognitive strategies to challenge dysfunctional beliefs associated with fear of flying (VRET); (b) computer-aided exposure with a therapist present throughout the exposure sessions but only solving the possible doubts that could appear using the system and not directly involved in the therapeutic process (CAE-T); and (c) a totally self-administered computer-aided exposure (CAE-SA). The study builds on the advances in research on VR and computer-aided psychotherapy, as described in previous works (Botella et al., 2007; Botella et al., 2008).

To our knowledge, no previous studies have directly compared VRET and CAE, though other trials have shown that the treatment outcomes for both interventions for flying phobia are quite similar (Bornas et al., 2006). However, a direct comparison is required; specially because the procedures differ in the kind of therapist involvement required and in the cost of devices used. Along these lines, this study could shed light on such topics as therapist involvement and, indirectly, cost-effectiveness. We also compared application of CAE with continuous therapist contact with a self-administered version in order to determine whether therapist involvement can be decreased during computer-based treatments without compromising efficacy. 
The study was based on the following hypotheses: (a) VRET and both of the CAE treatments would significantly reduce fear of flying before versus after the treatments, and that these gains would be largely maintained at follow-up; (b) results from applying the three treatment conditions would not differ significantly regarding the reduction of fear of flying and flying behavior both at post-treatment and at followup; and, (c) the highest attrition rates would be found in the self-administered procedure. The study also examined users' expectations and satisfaction with the various treatments, a topic on which the literature is not conclusive. The expectation was that (d) participants in the VRET condition would rank their treatment as being more valuable than that of the users of the CAE conditions, both at pretreatment and after treatment (since therapist involvement is substantially greater in VRET).

Expectations and satisfaction were expected to be high for all three conditions.

\author{
Method \\ Participants \\ Eighty-six individuals requested treatment; they were then screened for the
} study. Participants were recruited from the community through advertisements in local newspapers, flyers posted in our research centers and on the research group's website. Sixty participants met the criteria for the study and were randomly assigned to one of the three treatment conditions.

In order to be included in the trial, each participant had to meet current DSM-IV criteria for specific phobia (situational, related to fear of flying). Exclusion criteria included: people younger than 18, current psychological treatment, psychotropic medication use, any other current psychopathological disorder requiring immediate treatment, mental retardation, cardiovascular or respiratory illness, and current pregnancy. Twenty-six subjects were excluded from the study after the initial screening. 
Reasons for exclusion included fear of flying that does not meet criteria for specific phobia $(n=1)$, taking anxiolitic or antidepressive medication $(n=12)$, other axis I main diagnoses (panic disorder, major depressive disorder) $(n=7)$, chronic organic diseases $(n=2)$, developmental disabilities $(n=1)$, being under 18 years old $(n=2)$ and pregnancy $(n=1)$.

Demographic information, baseline scores on primary outcome measures, and time avoiding flying of enrolled participants are depicted in Table 1. None of them have previously received psychological treatment for fear of flying.

Insert table 1 about here

\section{Measures}

Diagnostic Status and Fear of Flying Features. The Anxiety Disorders Interview Schedule for DSM-IV (ADIS-IV; Brown, DiNardo, \& Barlow, 1994) was used at pretreatment and at post treatment to determine diagnostic status and to quantify levels of fear, avoidance, and interference on a scale of 0 to $8(0=$ no fear, avoidance or interference, $8=$ extreme fear, avoidance or interference). Specifically, the section on specific phobias of the Anxiety Disorders Interview Schedule for DMS-IV was used. ADIS-IV is an excellent interview for assessing anxiety disorders; it has proven adequate psychometric properties according to Anthony, Orsillo, and Roemer (2001).

Self-reported fear of flying. The Fear of Flying Questionnaire (FFQ; Bornas, Tortella-Feliu, García de la Banda, Fullana, \& Llabrés, 1999) and the Fear of Flying Scale (FFS; Haug et al., 1987) were used to assess self-reported of fear of flying. The FFQ is a 30 -item self-report instrument describing situations related to flying: anxiety during flight, anxiety experienced getting on the plane, and anxiety experienced by the 
observation of neutral or unpleasant flying-related situations. For each item, respondents rated their degree of discomfort associated with the situation on a scale of 1 to $9(1=$ not at all, $9=$ very much $)$. Scores ranged from 30 to 270 . As reported by Bornas et al. (1999), internal consistency was $\alpha=.97$ and retest reliability (15-day retest period) was $r=.92$. In our sample, Cronbach's alpha for the FFQ was .90 .

The FFS consists of 21 items describing air travel situations. Fear elicited by each situation was rated on a five-point scale $(0=$ not at all, $4=$ very much $)$, with scores ranging from 0 to 84. In the original FFS (Haug et al., 1987) Cronbach's $\alpha$ was .94 and retest reliability (at three months) was $r=.86$. For the translated version used in this study, Cronbach's $\alpha$ and 15-day retest reliability were $.95(n=228)$ and $.86(n=106)$ respectively (unpublished results). In our sample, Cronbrach's alpha for the FFS was .88 .

Clinician ratings. At the end of individual interviews, independent assessors rated, both at baseline and at post-treatment, the severity of the patients' phobias on a scale from 0 to 8 , where $0=$ symptom free and $8=$ extremely severe and disabling, for all aspects of life affected. This scale was the same as that used by Öst, Stridh, and Wold (1998). At the end of the interview the clinician rated the severity of the patient's phobia on a $0-8$ scale

Treatment expectations and satisfaction. Treatment expectations and satisfaction were measured after the treatment was presented in the first session, and also at posttreatment and at follow-up, using an adaptation of the Borkovec and Nau (1972) “Credibility/Expectation Scales". Several aspects were quantified, including: credibility (“To what extent does the treatment seem logical to you?"), usefulness (“To what extent do you think that the treatment is or was useful in your case?"), satisfaction (“To what extent are you satisfied with treatment (to be received or received)?”) and treatment 
aversion ("To what extent is or was the treatment aversive for you?"). Questions were answered on a scale of 0 to $10(0=$ not at all, $10=$ maximum $)$. This instrument has demonstrated good factorial structure, internal consistency and reliability according to Devilly and Borkovec (2000).

\section{Procedure}

All individuals who requested treatment were screened through a personal interview to determine their diagnostic status and to quantify the degree of fear, avoidance, and interference associated with their fear of flying. Also, fear of flying questionnaires (FFS and FFQ) were administered in the same session. All of the assessments were carried out by independent evaluators who were not involved in the randomization process. At the end of the screening, assessors rated their impressions on the severity of fear of flying. Suitable candidates for treatment $(n=60)$ were randomized to one of three treatment conditions: computer-aided exposure treatment assisted by a therapist (CAE-T) $(n=20)$, self-administered computer-aided exposure $(\mathrm{CAE}-\mathrm{SA})(\mathrm{n}=21)$ and virtual reality exposure treatment (VRET) $(\mathrm{n}=19)$. To control for procedural fidelity, detailed session-by-session therapist manuals were used. All of the eligible patients accepted treatment and signed an informed consent form.

\section{Treatments and Apparatus}

In the first session, for all three treatment conditions, we presented a 30 minute explanation of the mechanism contributing to the origin and maintenance of fear of flying; the rationale and structure of treatment were also provided. The goal was to briefly explain the following concepts: the role of avoidance in maintaining a phobia, other manifestations of phobic disorders (psychophysiological responses and cognitive concomitants), which is it prevalence, factors associated to the onset of fear of flying, the definition of exposure therapy, and methods for planning graded exposure using 
computer-based programs. This explanation was identical for all three experimental conditions, differing only in how the computer-based programs (VRET or CAE) work and how they would be used. A five-page booklet with explanations of fear of flying and treatment rationale was given to all participants. Before beginning exposure, the patients were familiarized with the computer programs and devices. Then, patients were asked about their satisfaction with the treatment rationale, with receiving the treatment, and their expectations for possible treatment results. Finally, exposure was conducted in the initial session under the unique conditions for each treatment.

VRET. The Virtual Flight software was used to provide exposure to three virtual scenarios: packing at home, waiting for boarding at the airport terminal, and sitting in the airplane while taking off and during flight. A detailed description of the virtual environments can be found in Botella et al. (2004). The software includes three VR scenarios: the room, the airport, and the plane.

The room is a scenario designed to address anticipatory anxiety. The patient is in a bedroom and he/she can perform some activities usually associated with the days or hours before a flight: packing, listening to news regarding air traffic and weather conditions. In the airport scenario the patient can listen to flight announcements and see them on the board, knowing that his/her flight is approaching; he/she can listen to people talking about flying; it is also possible to see and hear planes taking off and landing; finally the patient can arrive at the gate, and enter the plane. In the plane scenario, the patient is seated close to the window and can listen to the radio or read a magazine while waiting for the plane to start its engines. The flight process includes watching the safety demonstration, hearing the captain welcoming the passengers, takeoff and flight (in which the therapist can change the weather conditions, generate thunder, lighting, and turbulence), and landing. 
During exposure, the participant sat on a standard office chair and wore a 5DT HDM 800 immersive head mounted display attached to a personal computer (Intel Core 2 Duo E6850, 3 GHz, 2MB RAM; graphic engine ASUS Extreme AX300SE/T 128 $\mathrm{MB})$. The virtual reality equipment was operated by the therapist.

The main component of the treatment was VR exposure that included some kind of cognitive restructuring. Except for the first session (as described in the Procedure section), the following sessions were devoted to virtual exposure to the different scenarios, progressing from the easiest to the most difficult situations (according to the participant hierarchy established by the therapist with the patient in the first session along with data from the pretreatment assessment). The main goal of each exposure was for the patient to remain in the situation until a significant decrease in subjective anxiety was achieved. Therapists attended all VRET sessions, applying the VRET system and guiding the use of cognitive strategies. Every five minutes the therapists asked the patients to rate their anxiety levels and to verbalize the catastrophic thoughts and feelings experienced during exposure. The therapists challenged the dysfunctional beliefs associated with fear of flying. Patients did not receive homework, and practicing in vivo between sessions was not encouraged.

$C A E-T$. CAE was conducted with CAFFT2 online software displayed on a personal computer (Pentium IV 2.80GHz 512 MB RAM) connected to the Internet and with a 17-inch screen (graphic engine radeon 7000AGP 64MB). The CAFFT2 automatically configured a display of photos and associated sounds according to the patient's fear hierarchy for flight-related situations (drawn from answers to the Fear of Flying Questionnaire). Six sequences from 1 minute 51 seconds to 2 minutes 49 seconds were included: buying a ticket, packing at home, going to the airport and boarding, taking off, flying, landing, and hearing news about an aircraft accident. At the 
end of each sequence, the program requested fear ratings; based on the replies, the exposure continued with the same sequence or proceeded to the next sequence in the hierarchy. The CAFFT2 has been fully described elsewhere (Bornas et al., 2001; Bornas et al., 2006; Bornas et al., 2002). Therapists attended all CAE-T sessions, although they were instructed not to intervene unless technical problems occurred. If patients asked about the treatment process, they were invited to utilize the program's online help system.

$C A E-S A$. The CAE was also conducted with CAFFT2 online as described for the CAE-T group. However, the CAE-SA treatment was essentially self-administered; face-to-face therapist support was available only during the first and last sessions. At the first session, the information and instructions for using the computer program were the same as for CAE-T; however, they also informed the patients that they would undergo exposure alone with the computer. Additionally, they learned that their progress would be monitored; the online computer system allowed the therapist to know when the patient accessed the program, the duration of the sessions, the number of exposure trials completed, discomfort rates after each exposure, and so on. A telephone number was provided for contacting the therapist during the exposure sessions in case problems occurred with the computer or with the procedure. The therapists and users scheduled five additional days for one-hour exposure sessions with the computer in the university clinic. The first exposure trials were begun with a therapist present, to make sure the patients had completely understood the instructions. Then, the therapist left the clinic while the user was exposed to images and sounds. In the following sessions the user simply asked the receptionist for access to the session room. At the last session, each patient had a personal 20 minute meeting with a therapist. Because the therapists tracked the patients' progress, they could predict on which day the treatment would 
likely end. The meetings at the conclusion of the last session were designed to review how the treatment worked and to encourage the patients to take a real flight on their own in the following 15 days. Patients also answered two self-report measures on fear of flying.

For each treatment, a maximum of six one-hour, twice-weekly sessions was established. However, participants could terminate treatment before the sixth session if they completed all of the treatment modules. All treatments were conducted in the University Clinic Laboratories. All individuals who started treatment paid 90 Euros. At the conclusion of the treatments, patients were encouraged to take a flight on their own without any therapeutic help in the following 15 days. In the last session, patients completed fear of flying questionnaires and scheduled an interview for approximately 15 days later. Posttreatment and one-year follow-up interviews were conducted (independent of whether the patient had flown or not) by an independent assessor unaware of the participants' treatment conditions, to determine the diagnostic status, the present degree of fear, avoidance, and interference associated with fear of flying and the external rating of severity. They also asked at posttreatment and at one-year follow-up about participants' satisfaction with the treatment they received.

\section{Statistical analyses}

Analyses included an intent-to-treat (ITT) using the LVCF (last value carried forward) method. Missing values at post-treatment and/or at one-year follow-up were imputed with the participant's pretreatment score. This method was selected to minimize any potential inflation of post treatment results. In some cases, data were reported only for participants who completed treatment. To assess treatment effects, all outcome measures with continuous data were analyzed with mixed analyses of variance (general linear model), with group as the between-subject factor (CAE-T, CAE-S, 
VRET) and time as the within-subject factor (pretreatment, posttreatment, one-year follow-up). The within-group effect sizes of change were analyzed within and groups using Cohen's $d$ (Hedges corrected).

The Mann-Whitney $U$ test was used to determine possible differences among participants who dropped out or did not attend the posttreatment and/or follow-up assessments and those who finished treatment and attended all assessment sessions. At baseline, treatment groups were compared through one-way analyses of variance or chisquare tests, to determine if randomization was successful and to explore possible differences in treatment credibility or expectations.

Chi-square tests were performed to evaluate group differences for participants taking an actual flight after treatment and during the follow-up period, clinically significant improvement rates based on Jacobson and Truax (1991) indexes for FFQ scores, and number of participants free of specific phobia diagnosis related to fear of flying both at posttreatment and at one-year follow-up.

Results

\section{Equivalence of groups}

As depicted in Table 1, no significant differences were found before treatment among participants in the three treatment conditions, socio-demographic variables, or fear of flying measures. It can thus be concluded that randomization was successful.

\section{Attrition}

One participant withdrew from each of the following groups: CAE-T (5\%) and VRET (5.26\%). Both alleged scheduling problems after not attending scheduled sessions. Two participants withdrew from CAE-SA (9.5\%); one also reported scheduling conflicts after not attending a session and the other one felt ill after treatment 
began. No significant differences were found among treatment conditions in attrition rates $\left(\chi^{2}(2)=0.425, p=.809\right)$.

Participants who dropped out did not differ from participants who completed the treatment in any pretreatment variable except for treatment expectations $(p=.017)$; those who withdrew scored significantly lower $(M=4.50, S D$ 3.00, $U$ Mann-Whitney mean rank 11.12) than those who completed the sessions $(M=7.89, S D 1.41)(U$ MannWhitney mean rank 31.88).

Three participants who completed treatment, one per intervention group, did not attend the two week posttreatment assessment session. They did not differ from participants attending the 15-day posttreatment assessment in any of the measured variables.

At one-year follow-up, 38 participants attended the assessment session $(63.33 \%$ of those who started treatment, $71.70 \%$ of those who finished treatment and completed posttreatmemt assessment). No significant differences were found among treatment conditions at one-year follow-up attendance $\left(\chi^{2}(2)=0.730, p=.694\right)$. The fifteen participants who did not attend follow-up did not differ from those who did in any of the posttreatment variables except for avoidance as measured by ADIS-IV; those who did not attend follow-up assessment scored significantly higher $(M=3.93, S D 3.08)$ than those who did $(M=2.03, S D 2.65)(t(51)=2.26, p=.028)$.

Treatment effects on self-reported fear of flying, interview and clinicians' ratings.

As depicted in Table 2, results of an intent-to-treat analysis (last value carried forward method) indicate that time main effects were significant for all fear of flying self-report measures, main ratings in ADIS-IV, and in clinicians' ratings on the severity of the patients' phobia, revealing overall treatment effect. Contrasts show a significant reduction from pretreatment to posttreatment and from pretreatment to one-year follow- 
up in all of the fear of flying variables. From posttreatment to one-year follow-up, decreases in self-reported fear of flying remained stable, whereas fear, avoidance, and interference ratings in the ADIS-IV as well as clinicians' ratings on the severity of the patients' phobia worsened. However, reductions from pretreatment to one-year followup are clearly statistically and clinically significant, as shown in the pairwise comparisons and standardized effect sizes reported in Table 2.

Insert table 2 about here

Treatment x group interaction effects were not significant for any variables, indicating no different treatment effects. Likewise, group main effects were not significant for any of the dependent variables; that is, treatment groups did not differ significantly from each other. All three conditions exhibited significant effects for all outcome measures from both pretreatment to posttreatment and from pretreatment to one-year follow up.

\section{Clinically meaningful improvement and diagnostic status at posttreament}

Clinically meaningful improvement was calculated for FFQ scores using the Jacobson and Truax's (1991) indexes. As shown in Table 3, both at posttreatment and at follow-up, most of the participants fell into the category of "recovered" or "improved" regardless of their treatment conditions, with no statistically significant differences among treatments; this is true both for the completers' and for the ITT analyses.

Insert table 3 about here 
Posttreatment interviews revealed that the majority of participants were specific phobia (situational) diagnosis-free (Table 3) with no differences among groups for diagnostic status. At one-year follow-up, the groups did not differ regarding diagnostic status for each condition. However, percentage of phobia-free participants in all three treatment groups decreased to $50 \%$ at the one-year assessment.

\section{Behavioral outcomes}

At the end of treatment, participants were encouraged to take a real flight on their own as soon as possible. Two weeks after the last treatment session, they were contacted to determine if they had done so, and to schedule the posttreatment interview. During this 15 -day period, 10 out of 18 participants who had completed treatment in the CAE-T group flew (50\% of CAE-T participants), compared to 13 out of 18 in the CAESA group (61.9\% of CAE-SA participants), and 15 out of 17 in the VRET group $(78.9 \%$ of VRET participants). No differences were found among groups nor among those who had completed treatment $\left(\chi^{2}(2)=4.60, p=.100\right)$ nor in the ITT analysis $\left(\chi^{2}(2)=3.54, p\right.$ $=.170)$.

From posttreatment to the one-year follow-up assessment, 9 out of 14 participants in the CAE-T group who attended the assessment sessions flew (45\% of CAE-T participants enrolled in this group; number of flights $M=3.43$, $s d=6.34$ ), compared to 8 out of 12 both in the CAE-SA group (38.1\% of those who started treatment) and in the VRET group (36.8\% of those who started treatment). The mean number of flights taken by CAE-SA participants was $M=2.73, s d=3.61$, and the mean was $M=1.83, s d=1.80$ for VRET participants. No differences in taking or not taking a flight were found among groups, nor among those who attended follow-up $\left(\chi^{2}(2)=\right.$ $0.22, \mathrm{p}=.989)$ nor in the ITT analysis $\left(\chi^{2}(2)=0.69 \mathrm{p}=.708\right)$. Groups did not differ statistically in the number of flights taken $(F(2,36)=0.406, p=.670)$. 
Treatment features and treatment satisfaction ratings.

As stated in the Procedure section, all treatments comprised a maximum of six one-hour, twice-weekly sessions. Despite pre-established length of the treatments, an individual participant's treatment could be shortened if the participants completed the last exposure sequence in their own hierarchy before the sixth session. Indeed, some participants achieved this. The mean number of sessions for each treatment was $M=$ $4.70(S D$ 1.34) in the CAE-T, $M=4.10(S D$ 1.51) in the CAE-SA, and $M=5.32(S D$ 1.42) in the VRET. One-way ANOVA revealed statistically significant differences among groups $(F[2]=3.65, p=.032)$. Post-hoc analyses indicated that the CAE-SA group differed significantly from the VRET group $(t[1]=2.70, p=.032)$, but did not differ significantly from the CAE-T group $(p=.405)$; the same was true for the CAE-T group as compared to the VRET group $(p=.410)$.

As described in the Procedure section, after the treatment to be used was presented during the first treatment session, patients were asked (with each question rated on a scale of 0 to 10 ) about treatment credibility, satisfaction with receiving the treatment, expectations for possible treatment results (usefulness), and expected aversiveness of the treatment. Participants in all three conditions generally evaluated treatments as logical, satisfactory, useful and not very aversive. Treatment groups differed in the credibility granted to their respective interventions. Multiple comparisons revealed significant differences between the VRET and CAE-SA groups $(F=3.12 ; p=.011)$, while there were no differences between the VRET and CAE-T groups and CAE-T and CAE-SA groups. A similar though not statistically significant tendency was observed for expectations for treatment results (usefulness and satisfaction). 
At the end of treatment and at follow-up, participants were again asked for their opinions on the treatment rationale (treatment credibility), satisfaction with the treatment, the treatment usefulness in their particular cases, and whether the treatment had been aversive. At the end of treatment, participants in all three conditions generally evaluated treatments as very logical (credible), satisfactory, useful, and not very aversive. Virtual reality was, in all cases, the preferred treatment. Statistically significant differences among groups were found in approval of the treatment rationale, and in satisfaction with treatment. Multiple comparisons revealed that participants in the VRET group approved of the treatment rationale more than participants in the CAE-SA group $(F=2.99, p=.016)$. No statistically significant differences were found between the VRET and CAE-T groups, nor between the CAE-T and CAE-SA groups for this variable. Multiple comparisons also showed that participants in the VRET group were more satisfied with treatment than those in the CAE-T $(F=2.53, p=.049)$ and CAESA $(F=4.31, p=.0001)$ groups. The CAE-SA and CAE-T groups did not differ significantly in any of these participant ratings. No statistically significant differences emerged among groups in evaluations of treatment usefulness or aversive aspects of the interventions.

At one-year follow-up, previous differences among groups for expectations or satisfaction with treatment completely disappeared, and no differences were found among groups for any of these variables.

\section{Discussion}

To the best of our knowledge, this is the first study comparing VRET with other forms of computer-based treatment. In this trial, VRET was compared with computeraided exposure treatment as assisted by a therapist or self-administered. As 
hypothesized, all three treatment conditions were equally effective at significantly reducing fear of flying statistically and clinically, both at post-treatment and at one-year follow-up, as measured by the following: self-reported fear, number of participants taking a flight on their own during a 15-day period after treatment and during the following year, fear ratings, degree of avoidance and interference in the ADIS-IV interview, severity as assessed by independent assessors' ratings, and number of participants becoming free of diagnosis for specific phobia (situational). Nevertheless, it must be noted that reductions in fear at post-treatment were not fully maintained at oneyear follow-up. After one year, ADIS-IV main ratings (for fear, avoidance, and interference), and clinical ratings for global severity, excluding self-reported fear of flying measure worsened significantly; however, they remained significantly lower than pretreatment ratings.

As expected, large within-group effect sizes were found for all three treatment conditions from pretreatment to post-treatment, comparable to those reported in previous studies for VRET (Parsons \& Rizzo, 2008). At one-year follow-up, withingroup effect sizes remained large for all treatment conditions, though smaller than at post-treatment.

Neither at post-treatment nor at follow-up were there statistically significant differences among treatment conditions in clinically meaningful improvement, diagnostic status, or behavioral outcomes (including taking or not taking a flight and number of flights taken). Most participants were categorized as "recovered" or “improved" at both assessment times. Regarding diagnostic status, most patients were specific phobia (situational) diagnosis-free at post-treatment; however, at one-year follow-up there was a decline in the number of participants without a specific phobia (situational) diagnosis. Just as most of the participants in all groups had taken a flight on 
their own at post-treatment, most participants who attended one-year follow-up also flew during the following year.

Contrary to our third hypothesis (that the highest attrition rates would be found in the self-administered procedure), no greater attrition was observed in the CAE-SA group than in the other two treatment conditions; furthermore, no single participant refused treatment. Marks and Cavanagh (2009) posited that perhaps only a few minutes of formal contact with a therapist might be sufficient to prevent discontinuation. Sufficient confidence in the program was perhaps ensured through some or all of the following measures: personal initial and final session meetings, the availability of phone and e-mail contact with therapists (though this was not used by any patient), and informing participants that their progress in the CAE was tracked through online data that was relayed to a therapist.

The last aim of our study was to analyze users' expectations and satisfaction with the various treatments. This is a controversial topic on which the literature is not conclusive (e.g., Whitfield \& Williams, 2004; Cavanagh et al., 2009). We hypothesized that expectations and satisfaction would be high for all three treatment conditions. In the fourth hypothesis we stated that because therapist involvement is substantially greater in the VRET treatment condition, participants in that group would rank their treatment as more valuable than users of the CAE conditions. Our results yielded the following conclusions. First, both expectations at pretreatment and satisfaction were high for all treatment conditions, although they decreased at one-year follow-up. This is probably related to the reduced gains from post-treatment to follow-up. Second, as hypothesized, participants in VRET condition had greater expectations for treatment before starting it, mainly in terms of credibility, and they were also more satisfied with the intervention at 
post-treatment compared to the CAE groups. However, these differences disappeared at follow-up; groups did not diverge in any of these variables.

Our results raise some interesting questions to be further explored. This is not the first study indicating that treatment effects are not enhanced by enriching computergenerated exposure environments to appear more realistic. Mühlberger et al. (2003) showed that adding motion and vibration simulations did not further enhance the treatment effects of VRET for visual and acoustic stimuli. Development of CAFFT2 also demonstrated that more sophisticated immersive conditions (such as darkness and projecting large screen images) did not produce better results than exposure to images and sounds on a standard PC screen in a lighted room (Bornas et al., 2001; Bornas et al., 2006). These data suggest that less sophisticated and cheaper devices might be sufficient for producing satisfactory outcomes, at least in some cases. Furthermore, these results emphasize the need for further investigation into the processes that can provoke the subjective sensation of "being there" in the mediated environment, as well as the role of this sense of "presence" in therapeutic efficacy. Interestingly, it may be that the similar outcomes for VRET and CAE in our study can be partially attributed to the critical role of sound in evoking anxiety in patients who exhibit fear of flying (Bornas et al., 2004). Sound might be more relevant than the visual cues that are addressed by other immersive conditions or the interaction provided by virtual reality. In other words, equivalence of virtual reality and other computer-aided systems for providing successful exposure treatment for fear of flying may not be generalizable to different anxiety disorders where visual cues are more significant than auditory ones. It is interesting to note that the computer-aided exposure treatments with minimal therapist participation (mainly in the self-administered condition) obtained equivalent outcomes as VRET, despite the fact that VRET was applied with a therapist playing a 
central role (elaborating the hierarchy, controlling the computer inputs and especially by applying some cognitive strategies). This has obvious efficiency implications, as does the fact that CAE treatments were completed in fewer sessions than VRET. That is, if our results are replicated, in a stepped care model, self-administered CAE treatments for fear of flying could be recommended before opting for more costly procedures that require greater therapist involvement such as VRET or CAE which require continuous therapist assistance.

Another feature of the study worthy of additional attention is to determine the true weight of cognitive strategies in VRET. In the present trial, the use of cognitive strategies was minimal compared to other VRET studies wherein some sessions are devoted specifically to cognitive issues. However, there was no "pure exposure" with any cognitive component, as there was in both CAE groups. Krijn et al. (2007) partially concluded from the results of their study that because VRET (as the sole component of intervention) was not sufficiently effective at reducing fear of flying, perhaps a combination of techniques (e.g. cognitive restructuring) was necessary. They state the need for future research on direct comparison of VRET and VRET plus cognitive techniques.

We agree that this is necessary, but for a different reason. Our belief is that therapist-guided cognitive restructuring is not in fact central to exposure treatment effects, as the results obtained in this study of CAE treatments suggest. This alternative hypothesis would lead us to believe that it is possible to design new VR programs in the form of navigable scenarios which would enable self-administration of VRET for fear of flying (with or without formal and therapist-guided cognitive restructuring). This technological development could enable the dissemination of VR systems to interventions without requiring continuous personal therapist support, thus saving 
therapist time. This is not to imply that the role of the therapist is insignificant, nor that cognitive strategies are useless; rather, the point is that VRET can perhaps be administered with less therapist involvement and making these kinds of applications more accessible to the general population without reducing their efficacy. The results of the present trial support the hypothesis that both the VRET system and the presence of the therapist in CAE-T increase the user's satisfaction and/or expectations for treatment, at least in the short term; additionally, they reduce the aversiveness of the intervention to some extent, although not to statistically significant levels. Future studies should explore which kinds of treatment conditions are most compatible with patient characteristics in order to maximize the intervention's efficiency.

The main limitation of our study is the relatively small number of participants in each treatment condition, which clearly compromises the statistical import of our results. Nevertheless, the number of subjects included in our study is not dramatically smaller than in other computer-based exposure treatment studies. To our knowledge, the largest sample in a fear of flying treatment study using virtual reality included 83 enrolled participants and 75 completers (Rothbaum et al., 2006). In the majority of these kinds of studies, the sample size is smaller than that of in the present trial (e.g. Rothbaum et al., 2000; Maltby et al., 2002; Mühlberger et al., 2001, 2003). Although our sample size was limited, the innovative approach of the trial is noteworthy, as well are its provocative results. At the very least, they serve as a point of comparison in these kinds of interventions.

Another limitation of the study is the absence of a behavioral avoidance test at the initial assessment. Behavioral avoidance tests for fear of flying are very difficult and costly to conduct. In fact, they are not included in most flight phobia treatment controlled trials. However, the degree of self-reported avoidance was very high in all 
groups, and no participants who presented only slight fears were included in the study. Therefore, we believe the lack of a behavioral avoidance test at baseline is not a serious shortcoming for the trial, although we must cautiously evaluate the results regarding the number of participants who flew at post-treatment.

A third limitation is the absence of a control group. Of course, including a control condition would have strengthened the current study, though there were two reasons this was not done. Firstly, previous studies comparing CAE treatment assisted by a therapist and VRET with control conditions have provided evidence about their superior efficacy with control groups. Furthermore, other researchers (e.g., Öst, 1997) have indicated that the absence of a control group without treatment is not a crucial methodological problem in studies examining the efficacy of treatments for specific phobias; this is due to the clear superiority of these treatments and the almost insignificant proportion of participants who naturally recover over time. Secondly, the inclusion of a control group in this particular study would have required us to further reduce the number of participants in each group.

Despite these limitations, results of our study shed light on certain topics that are central to the research and dissemination of computer-based exposure treatments and add valuable insights to the existing literature. 
Acknowledgments

Funding for the study was provided by grants SEJ2005-00899/PSIC, SEJ-200614301/PSIC, PROMETEO/2008/157, and CIBER Fisiopatologia de la Obesidad y Nutricion - ISCIII CB06/03/0052 from the Spanish Government. 


\section{References}

Andersson, G. (2009). Using the Internet to provide cognitive behaviour therapy. Behaviour Research and Therapy, 47, 175-180. doi: 10.1016/j.brat.2009.01.010

Anderson, P., Jacobs, C. H., \& Rothbaum, B. O. (2004). Computer-supported cognitive behavioral treatment of anxiety disorders. Journal of Clinical Psychology, 60, 253267. doi: $10.1002 /$ jclp. 10262

Anthony, M. M., Orsillo, S. M., \& Roemer, L. (2001). Practitioner's Guide to Empirically Based Measures of Anxiety. New York: Plenum.

Baños, R. M., Botella, C., Perpiñá, C., Alcañiz, M., Lozano, J. A., Osma, J, \& Gallardo, M. (2002) Virtual Reality Treatment of Flying Phobia. IEEE-Transactions on Information Technology in BioMedicine, 6, 206-212. doi:

10.1109/TITB.2002.802380

Borkovec, T. D., \& Nau, S. D. (1972). Credibility of analogue therapy rationales. Journal of Behaviour Therapy and Experimental Psychiatry, 3, 257-260. doi:10.1016/0005-7916(72)90045-6

Bornas, X., Fullana, M. A., Tortella-Feliu, M., Llabrés, J., \& García de la Banda, G. (2001). Computer-assisted therapy in the treatment of flight phobia: a case report. Cognitive and Behavioral Practice, 8, 234-240. doi:10.1016/S1077-7229(01)800584

Bornas, X., Llabrés, J., Noguera, M., López, A. M., Barceló, F., Tortella-Feliu, M., \& Fullana, M. A. (2004). Self-implication and heart rate variability during simulated exposure to flight-related stimuli. Anxiety, Stress, and Coping, 17, 331-339. doi: $10.1080 / 10615800512331328777$ 
Bornas, X., Tortella-Feliu, M., Fullana, M. A., \& Llabrés, J. (2001). Computer-assisted treatment of flight phobia: a controlled study. Psychotherapy Research, 11, 259-273. doi:10.1080/713663983

Bornas, X., Tortella-Feliu, M., García de la Banda, G., Fullana, M. A., \& Llabrés, J. (1999). Validación factorial del Cuestionario de Miedo a Volar [Factorial validation of the Fear of Flying Questionnaire]. Análisis y Modificación de Conducta, 25, 885907.

Bornas, X., Tortella-Feliu, M., \& Llabrés, J. (2006). Do all treatments work for flight phobia? Computer-assisted exposure versus a brief multicomponent nonexposure treatment. Psychotherapy Research, 16, 41-50. doi: 10.1080/10503300500091058

Bornas, X., Tortella-Feliu, M., Llabrés, J., Barceló, F., Pauli, P., \& Mülhberger, A. (2002). Clinical usefulness of a simulated exposure treatment for fear of flying. International Journal of Clinical and Health Psychology, 2, 247-262. Retrieved from http://www.aepc.es/ijchp/busca.php?coid=English

Botella, C., Osma, J., García-Palacios, A., Quero, S., \& Baños, R. M. (2004). Treatment of flying phobia using virtual reality: Data from a 1-year follow-up using multiple baseline design. Clinical Psychology and Psychotherapy, 11, 311-323. doi: 10.1002/cpp.404

Botella, C., Quero, S., Baños, R. M., García-Palacios, A., Bretón-López, J., Alcañiz, M., \& Fabregat, S. (2008). Telepsychology and Self-Help: The Treatment of Phobias Using the Internet. CyberPsychology and Behavior, 11, 659-664. doi:10.1089/cpb.2008.0012

Botella, C., Quero, S., Baños, R. M., Perpiñà, C., García-Palacios, A., \& Riva, G. (2006). Virtual reality and psychotherapy. In G. Riva, C. Botella, P. Légeron, \& G. Optale (Eds.), Cybertherapy. Internet and virtual reality as assessment and 
rehabilitation tools fro clinical psychology and neuroscience (pp. 37-52).

Amsterdam: IOS Press.

Bowers, W., Stuart , S., \& MacFarlane, R. (1993). Use of computer-administered cognitive- behavior therapy with depressed patients. Depression, 1, 294-299. doi: 10.1002/depr.3050010603

Brown, T. A., DiNardo, P. A., \& Barlow, D. H. (1994). Anxiety Disorders Interview Schedule for DSM-IV (ADIS-IV). Adults version. Albany, NY: Graywind Publications.

Cavanagh, K., Shapiro, D. A., Van Den Berg, S., Swain, S., Barkham, M. \& Proudfoot, J. (2009). The Acceptability of Computer-Aided Cognitive Behavioural Therapy: A Pragmatic Study. Cognitive Behaviour Therapy, 38, 235-246. doi $10.1080 / 16506070802561256$

Cuijpers, P., Marks, I., van Straten, A., Cavanagh, K., \& Andersson, G. (2009). Computer-aided psychotherapy for anxiety disorders: A meta-analytic review. Cognitive Behaviour Therapy, 38, 66-82. doi: 10.1080/16506070802694776

Devilly, G. J., \& Borkovec, T. D. (2000). Psychometric properties of the credibility/expectancy questionnaire. Journal of Behavior Therapy, 31, 73-86. doi:10.1016/S0005-7916(00)00012-4

Graham, C., Franses, A., Kenwright, M., \& Marks, I. M. (2000). Psychotherapy by computer: A postal survey of responders to a teletext article. Psychiatric Bulletin, 24, 331-332. doi: 10.1192/pb.24.9.331

Haug, T., Brenne, L., Johnsen, D. H., Brentzen, D., Götestam, K. G., \& Hughdal, K. (1987). A three systems analysis of fear of flying: A comparison of a consonant versus a non-consonant treatment method. Behaviour Research and Therapy, 25, 187-194. doi:10.1016/0005-7967(87)90045-3 
Jacobson, N. S., \& Truax, P. (1991). Clinical significance: a statistical approach to defining meaningful change in psychoterapy research. Journal of Consulting and Clinical Psychology, 59, 12-19. Retrieved from

http://www.sciencedirect.com/science/journal/0022006X

Kaltenthaler, E., Parry, G., \& Beverley, C. (2004). Computerized cognitive behaviour therapy: A systematic review. Behavioural and Cognitive Psychotherapy, 32, 31-55. doi:10.1017/S135246580400102X

Krijn, M., Emmelkamp, P. M. G., Olafsson, R. P., \& Biemond, R. (2004). Virtual reality exposure therapy of anxiety disorders: A review. Clinical Psychology Review, 24, 259-281. doi:10.1016/j.cpr.2004.04.001

Krijn, M., Emmelkamp, P. M. G., Olafsson, R. P., Bouwman, M., van Gerwen, L. J., Spinhoven, P., ... van der Mast, C. A. P. G. (2007). Fear of flying treatment methods: Virtual reality exposure vs. cognitive behavioral therapy. Aviation, Space, and Environmental Medicine, 78, 121-128. Retrieved from http://www.ingentaconnect.com/content/asma/asem

Maltby, N., Kirsch, I., Mayers, M., \& Allen, G. J. (2002). Virtual reality exposure therapy for the treatment of fear of flying: a controlled investigation. Journal of Consulting and Clinical Psychology, 70, 1112-1118. Retrieved from http://www.sciencedirect.com/science/journal/0022006X

Marks, I., \& Cavanagh, K. (2009). Computer-aided psychological treatments (CP): Evolving issues. Annual Review of Clinical Psychology, 5, 163-183. doi: 10.1146/annurev.clinpsy.032408.153538

Marks, I. M., Cavanagh, K., \& Gega, L. (2007a). Hands-on Help. Computer-aided Psychotherapy. Hove: Psychology Press. 
Marks, I. M., Cavanagh, K., \& Gega, L. (2007b). Computer-aided psychotherapy: revolution or bubble? British Journal of Psychiatry, 191, 417-473. doi: 10.1192/bjp.bp.107.041152

Marks, I. M., Kenwright, M., McDonough, M., Whittaker, M., \& Mataix-Cols, D. (2004). Saving clinicians' time by delegating routine aspects of therapy to a computer: A RCT in phobia/panic disorder. Psychological Medicine, 34, 9-18. doi:10.1017/S003329170300878X

McCrone P., Marks I. M., Greist J. H., Baer L., Kobak K. A., Wenzel K.W., \& Hirsch M. J. (2007). Cost-effectiveness of computer-aided behaviour therapy for obsessivecompulsive disorder. Psychotherapy and Psychosomatics, 76, 249-250. doi:10.1159/000101504

Mühlberger, A., Herrmann, M., Wiedemann, G., Ellgring, H., \& Pauli, P. (2001). Treatment of fear of flying with exposure therapy in virtual reality. Behaviour Research and Therapy, 39, 1033-1050. doi:10.1016/S0005-7967(00)00076-0

Mühlberger, A., Weik, A., Pauli, P., \& Wiedemann, G. (2006). One-sessions virtual reality exposure treatment for fear of flying: 1-year follow-up and graduation flight accompaniment effects. Psychotherapy Research, 16, 26-40. doi: $10.1080 / 10503300500090944$

Mühlberger, A., Wiedemann, G., \& Pauli, P. (2003). Efficacy of a one-session virtual reality exposure treatment for fear of flying. Psychotherapy Research, 13, 323-336. dpi: $10.1093 / \mathrm{ptr} / \mathrm{kpg} 030$

Öst, L. G. (1997). Rapid treatment of specific phobias. In G. C. L. Davey (Ed.), Phobias. A handbook of theory, research and treatment (pp. 227-246). Chichester, UK: Wiley. 
Öst, L. G., Stridh, B. M., \& Wolf, M. (1998). A clinical study of spider phobia. Prediction of outcome after self-help and therapist-directed treatments. Behaviour Research and Therapy, 36, 17-35. doi:10.1016/S0005-7967(97)10018-3

Palmqvist, B., Carlbring, P., \& Andersson, G. (2007). Internet-delivered treatments with or without therapist input: does the therapist factor have implications for efficacy and cost?Expert Review of Pharmacoeconomics \& Outcomes Research, 7, 291-297. doi: $10.1586 / 14737167.7 .3 .291$

Parsons, T. D., \& Rizzo, A. A. (2008). Affective outcomes of virtual reality exposure therapy for anxiety and specific phobias: A meta-analysis. Journal of Behavior Therapy and Experimental Psychiatry, 39, 250-261. doi:10.1016/j.jbtep.2007.07.007

Powers, M. B., \& Emmelkamp, P. M. G. (2008). Virtual reality exposure therapy for anxiety disorders: A meta-analysis. Journal of Anxiety Disorders, 22, 561-569. doi:10.1016/j.janxdis.2007.04.006

Rothbaum, B. O., Anderson, P., Zimand, E., Hodges, L., Lang, D., \& Wilson, J. (2006). Virtual reality exposure therapy and standard (in vivo) exposure therapy in the treatment of fear of flying. Behavior Therapy, 37, 80-90. doi:10.1016/j.beth.2005.04.004

Rothbaum, B. O., Hodges, L., Smith, S., Lee, J. H., \& Price, L. (2000). A controlled study of virtual reality exposure therapy for the fear of flying. Journal of Consulting and Clinical Psychology, 68, 1020-1026. Retrieved from http://www.sciencedirect.com/science/journal/00057894

Wiederhold, B. K., \& Wiederhold, M. D. (2003). Three-year follow-up for virtual reality exposure for fear of flying. CyberPsychology and Behavior, 6, 441-445. doi:10.1089/109493103322278844 
Whitfield, G., \& Williams, C. (2004). If the evidence is so good—why doesn't anyone use them? A national survey of the use of computer-aided cognitive behaviour therapy. Behavioural and Cognitive Psychotherapy, 32, 57-65. doi:10.1017/S1352465804001031

Wolitzky-Taylor, K. B., Horowitz, J. D., Powers, M. B., \& Telch, M. J. (2008). Psychological approaches in the treatment of specific phobias: A meta-analysis. Clinical Psychology Review, 28, 1021-1037. doi:10.1016/j.cpr.2008.02.007 
Table 1.

Descriptive statistics of sample by treatment group

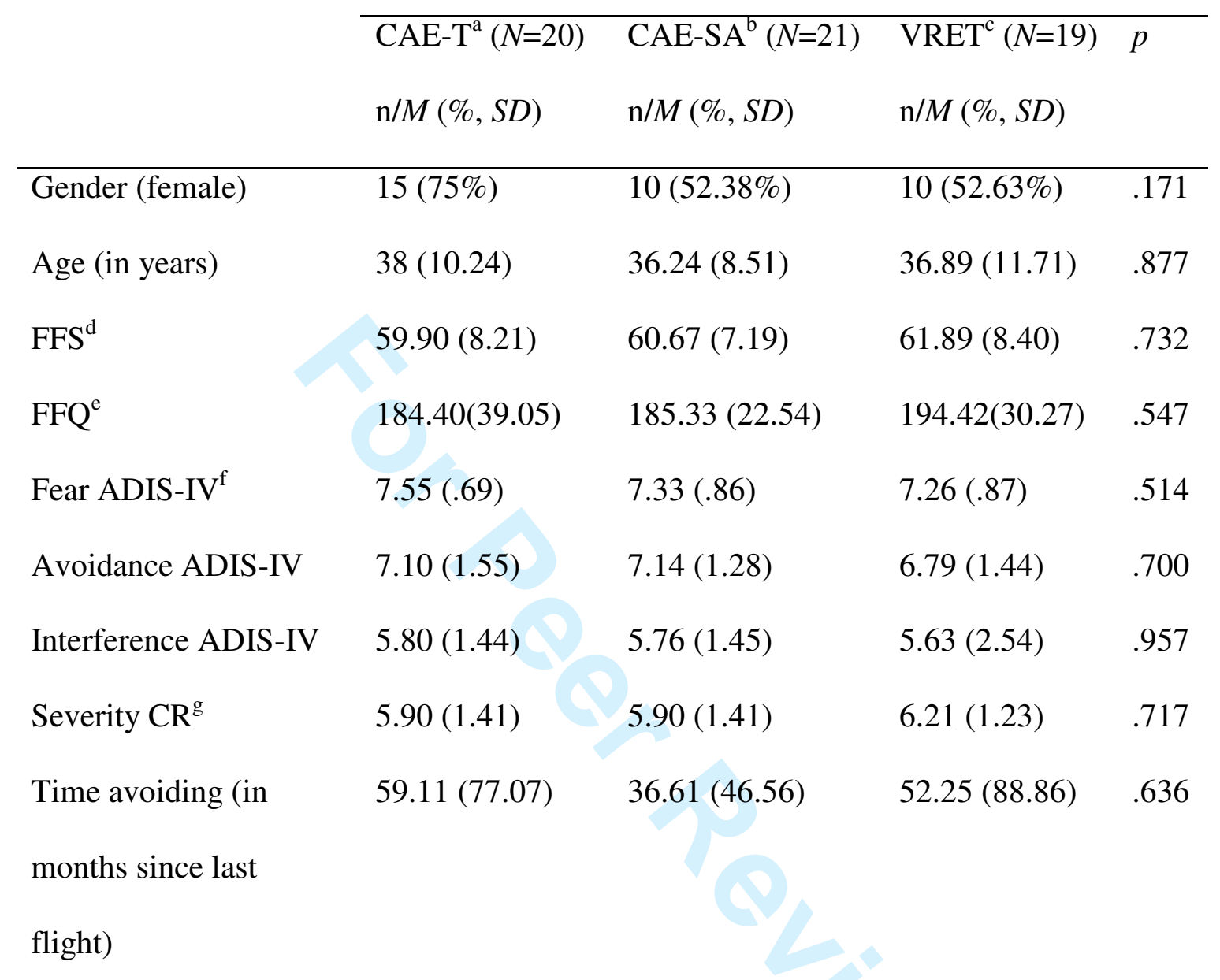

Computer-aided exposure with therapist assistance ${ }^{\mathrm{a}}$, Self-administered computer-aided exposure $^{\mathrm{b}}$; Virtual reality exposure treatment ${ }^{\mathrm{c}}$.

Fear of Flying Scale ${ }^{\mathrm{d}}$; Fear of Flying Questionnaire ${ }^{\mathrm{e}}$; Anxiety Disorders Interview Schedule for DSM-IV'; Clinician's rating ${ }^{\mathrm{g}}$ 
VR and CAE treatments 2

Table 2.

Means, standard deviations, and repeated measures analysis for effects of treatment at posttreament on self-reported fear of flying measures, interview data, and external evaluator intent-to-treat (ITT) analyses

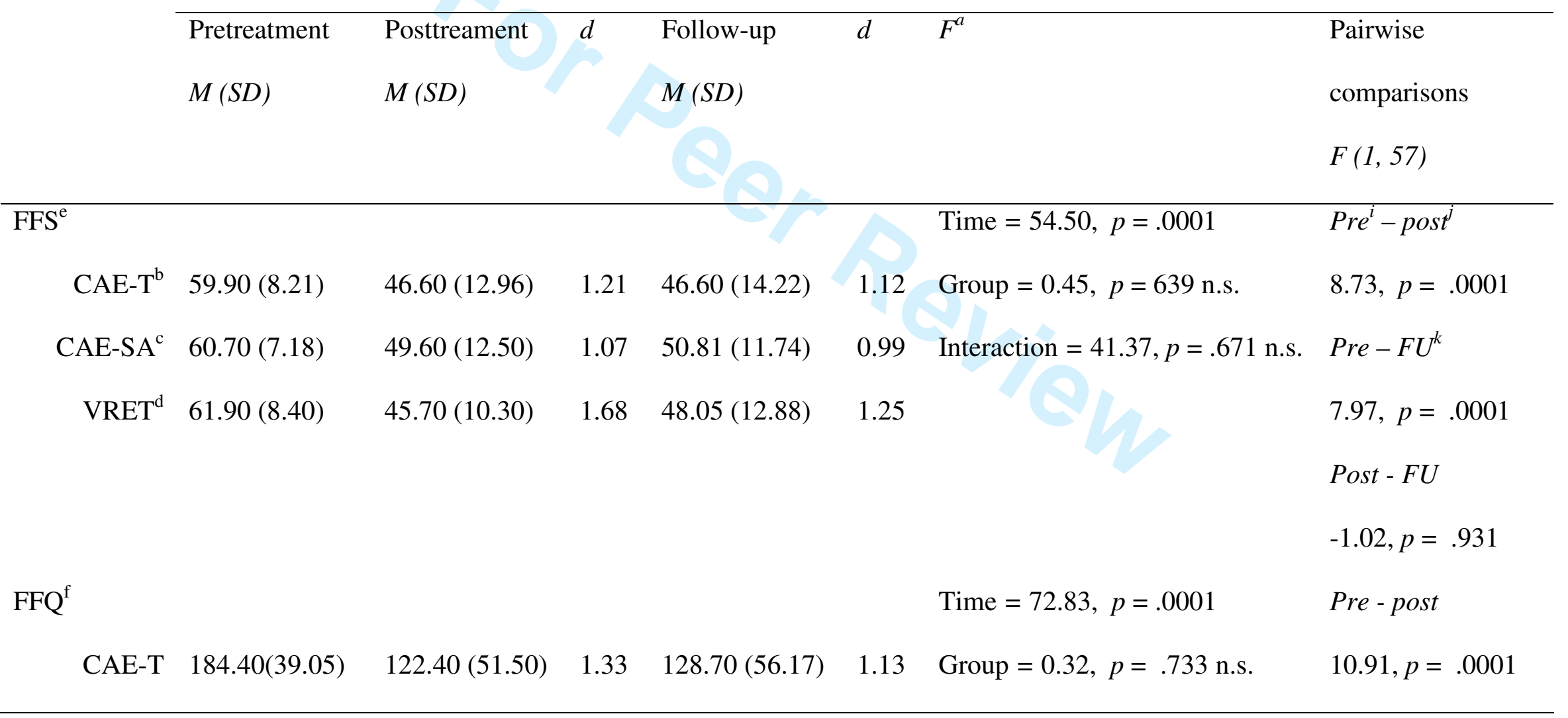


CAE-SA $185.33(22.54) \quad 129.43(38.00) \quad 1.76 \quad 133.67(45.87) \quad 1.40 \quad$ Interaction $=1.15, p=.337$ n.s. $\quad$ Pre $-F U$

$\begin{array}{llllll}\text { VRET } & 194.42(30.27) & 109.03(40.60) & 2.34 & 120.68(50.75) & 1.73\end{array}$

$8.25, p=.0001$

Post - FU

$-1.60, p=.344$

ADIS-IV ${ }^{\mathrm{g}}$

Fear

$\begin{array}{llllll} & & & & \text { Time }=72.42, p=.0001 & \text { Pre }- \text { post } \\ 4.00(2.55) & 1.86 & 5.00(2.39) & 1.42 & \text { Group }=0.21, p=.809 \text { n.s. } & 10.76, p=.0001 \\ 4.43(2.28) & 1.53 & 4.95(2.27) & 1.36 & \text { Interaction }=0.26, p=.902 \text { n.s. } & \text { Pre }-F U \\ 3.84(2.36) & 1.88 & 4.68(2.47) & 1.36 & 8,56, p=.0001\end{array}$

CAE-T 7.55 (.69)

CAE-SA $7.33(.86)$

VRET $7.26(.87)$

$3.84(2.36)$

$1.88 \quad 4.68(2.47) \quad 1.36$

Post - FU

$-0.26, p=.010$ 
VR and CAE treatments 4

\begin{tabular}{|c|c|c|c|c|c|c|c|}
\hline Avoidance & & & & & & Time $=63.60, p=.0001$ & Pre-post \\
\hline CAE-T & $7.10(1.55)$ & $3.05(3.35)$ & 1.52 & $4.45(3.27)$ & 1.02 & Group $=0.38, p=.683$ n.s. & $9.84, p=.0001$ \\
\hline CAE-SA & $7.14(1.28)$ & $3.57(3.19)$ & 1.44 & $4.43(2.98)$ & 1.16 & Interaction $=0.17, p=.953$ n.s. & Pre - FU \\
\hline VRET & $6.79(1.44)$ & $2.63(2.90)$ & 1.78 & $3.95(3.15)$ & 1.13 & & $\begin{array}{l}7.41, p=.0001 \\
\text { Post }-F U \\
-4.04, p=.0001\end{array}$ \\
\hline Interference & & & & & & Time $=45.50, p=.0001$ & Pre-post \\
\hline CAE-T & $5.80(1.44)$ & $2.50(2.19)$ & 1.75 & $3.90(2.34)$ & 0.96 & Group $=0.16, p=.854$ n.s. & $8.72, p=.0001$ \\
\hline CAE-SA & $5.76(1.45)$ & $3.19(2.44)$ & 1.26 & $3.29(2.37)$ & 1.23 & Interaction $=3.15, p=.945$ n.s. & Pre - FU \\
\hline VRET & $5.63(2.54)$ & $2.16(2.46)$ & 1.36 & $3.63(2.85)$ & 0.73 & & $\begin{array}{l}6.02, p=.0001 \\
\text { Post }-F U \\
-3.46, p=.003\end{array}$ \\
\hline Severity $\mathrm{CR}^{\mathrm{h}}$ & & & & & & Time $=58.36, p=.0001$ & Pre-post \\
\hline CAE-T & $5.90(1.41)$ & $3.10(2.45)$ & 1.37 & $4.10(2.42)$ & 0.89 & Group $=0.22, p=.805$ n.s. & $9.79, p=.0001$ \\
\hline CAE-SA & $5.90(1.41)$ & $3.48(2.52)$ & 1.16 & $3.86(2.63)$ & 0.95 & Interaction $=1.00, p=.408$ n.s. & Pre - FU \\
\hline
\end{tabular}


$\begin{array}{lllll}\text { VRET } & 6.21(1.23) \quad 2.63(2.06) & 2.07 \quad 3.37(2.73) \quad 1.31\end{array}$

Post - FU

$-2.77, p=.023$

Degrees of freedom and error for $F$ values: time $F(2,114)$, group $F(2,57)$, interaction $F(2,114)^{\text {a. }}$

Computer-aided exposure with therapist assistance ${ }^{\mathrm{b}}$, Self-administered computer-aided exposure ${ }^{\mathrm{c}}$; Virtual reality exposure treatment ${ }^{\mathrm{d}}$.

Fear of Flying Scale ${ }^{\mathrm{e}}$; Fear of Flying Questionnaire ${ }^{\mathrm{f}}$; Anxiety Disorders Interview Schedule for DSM-IV ${ }^{\mathrm{g}}$; Clinician's rating ${ }^{\mathrm{h}}$; Pretreatment ${ }^{\mathrm{i}}$;

Post-treatment ${ }^{\mathrm{j}}$; One-year follow-up ${ }^{\mathrm{k} .}$ 
Table 3.

Clinically meaningful improvement on the Fear of Flying Questionnaire (FFQ) scores and diagnostic status at post-treatment for those who completed treatment or attended one-year follow-up and intent-to-treat analyses

\begin{tabular}{llllll}
\hline \multicolumn{3}{c}{ At Postreatment } & \multicolumn{2}{c}{ At One-year Follow-up } \\
\hline CAE-T $^{\mathrm{a}}$ & CAE-SA $^{\mathrm{b}}$ & VRET $^{\mathrm{c}}$ & CAE-T $^{\mathrm{a}}$ & CAE-SA $^{\mathrm{b}}$ & VRET $^{\mathrm{c}}$ \\
$n(\%)$ & $n(\%)$ & $n(\%)$ & $n(\%)$ & $n(\%)$ & $n(\%)$
\end{tabular}

Clinical Improvement

Recovered

$\begin{array}{cccccc}\text { Completers } 9(47.4 \%) & 10(52.6 \%) & 12(66.7 \%) & 5(35.7 \%) & 4(33.3 \%) & 6(50 \%) \\ \text { ITT }^{\mathrm{d}} 9(45 \%) & 10(47.6 \%) & 12(63.2 \%) & 6(30 \%) & 8(38.1 \%) & 11(57.9 \%)\end{array}$

Improved

$\begin{array}{rlllll}\text { Completers } 7(36.8 \%) & 7(36.8 \%) & 6(33.3 \%) & 7(50 \%) & 6(50 \%) & 2(16.7 \%) \\ \text { ITT 7 (35\%) } & 7(33.30 \%) & 6(31.60 \%) & 10(50 \%) & 8(38.1 \%) & 3(15.8 \%)\end{array}$

No change
Completers $3(15.8 \%)$
$2(10.5 \%)$
$0(0 \%)$
$2(14.3 \%)$
$2(16.7 \%)$
$4(33.3 \%)$ 


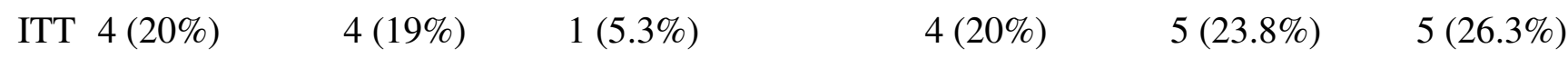

$$
\begin{aligned}
& \text { Completers }(\mathrm{n}=56): \chi^{2}(4)=3.37, p=.499 \quad \text { Completers }(\mathrm{n}=38): \chi^{2}(4)=4.08, p=.394 \\
& \operatorname{ITT}(\mathrm{n}=60): \chi^{2}(4)=2.54, p=.641 \quad \operatorname{ITT}(\mathrm{n}=60): \chi^{2}(4)=5.43 \%, p=.246
\end{aligned}
$$

Diagnostic Status

No diagnosis

$$
\begin{aligned}
& \begin{array}{llllll}
\text { Completers } & 12(66.7 \%) & 11(61.1 \%) & 13(76.5 \%) & 7(50 \%) & 6(50 \%)
\end{array} \\
& \begin{array}{llllll}
\text { ITT } 12(60 \%) & 11(52.4 \%) & 13(68.4 \%) & 8(40 \%) & 10(47.6 \%) & 10(52.6 \%)
\end{array} \\
& \text { Completers }(\mathrm{n}=56): \chi^{2}(2)=0.97, p=.617 \quad \text { Completers }(\mathrm{n}=38): \chi^{2}(2)=0.00, p=1.00 \\
& \operatorname{ITT}(\mathrm{n}=56): \chi^{2}(2)=1.07, p=.586 \quad \operatorname{ITT}(\mathrm{n}=60): \chi^{2}(2)=.636, p=.727
\end{aligned}
$$

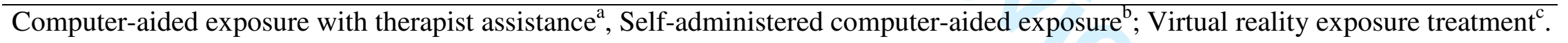

Intent-to-treat analysis ${ }^{\mathrm{d}}$ 
Dr. Miquel Tortella-Feliu is Senior Lecturer in Behavior Therapy at the Department of Psychology, University of the Balearic Islands (Majorca). His main research interests are anxiety disorders.

Dr. Cristina Botella is Full Professor of Clinical Psychology and Director of the Emotional Disorders Clinic at Jaume I University, Spain. Her main line of research is the design and testing of clinical applications based on information and communication technologies for the treatment of emotional disorders.

Dr. Jordi Llabrés is Senior Lecturer in Psychological Treatments at the Department of Psychology, University of the Balearic Islands (Majorca). His main research area is computer-aided cognitive-behavior therapy.

Dr. Juana María Bretón-López is a Lecturer (Assistant Professor) at the Universitat Jaume I, Spain. Her main research interests are anxiety disorders, especially phobic disorders and the application of new technologies to treat these. Bretón has a $\mathrm{PhD}$ in Clinical Psychology from the University of Granada.

Antonio Riera del Amo is a doctoral student at Jaume I University, Spain, where he is working in the design and testing of clinical applications based on information and communication technologies for the treatment of emotional disorders.

Dr. Rosa M. Baños is Full Professor of Psychopathology at the Universidad de Valencia, Spain. Her research interests include psychopathology, the treatment of psychological disorders, and the application of VR to Clinical Psychology. She has a $\mathrm{PhD}$ in Psychology from the University of Valencia.

Joan M. Gelabert is a doctoral student and clinical research assistant at the University of the Balearic Islands. He is also behavior therapist at USP Palmaplanas Hospital. His main research interests are computer-aided cognitive-behavior therapies. 